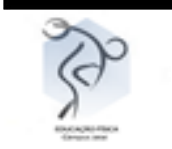

\title{
Treinamento esportivo: um estudo introdutório sobre suas bases científicas
}

\author{
Wanderson Pereira Lima ${ }^{1}$ \\ José dos Reis Júnior ${ }^{2}$ \\ João Pedro Barbosa Bandeira ${ }^{3}$
}

Resumo: o presente estudo tem como objetivo central apresentar uma discussão sobre o treinamento esportivo, enfatizando suas bases científicas. Este trabalho foi realizado mediante estudo bibliográfico em livros e artigos científicos especializados na área de Educação Física. Como resultados desses estudos foram apresentadas as principais características que constituem os princípios do treinamento esportivo, avaliação e controle, bem como, aspectos da iniciação a competição, ressaltando o treinamento de força para jovens, adultos e atletas. Conclui-se que trabalhar, objetivando a efetiva melhoria no desempenho esportivo da atleta e do não atleta, deve-se enfatizar o conhecimento científico, enfatizando a amplitude da área do treinamento esportivo, havendo assim mútua relação entre seus elementos básicos.

Palavras -chave: Treinamento esportivo. Conhecimento científico. Saúde.

\section{Sports training: an introductory study on its scientific basis}

Abstract:The present study presents a discussion about the sports training. It is emphasized its scientific bases. This study was carried out to the bibliographic study in books and scientific articles, specialized in the field of Physical Education. As a result of these studies were presented the main characteristics that constitute the principles of sports training, evaluation and control, as well as aspects of initiation to competition. It is highlighted strength training to youth, adults and athletes. Concluded about this study that working, aiming at the effective improvement in athlete's and athlete's athletic performance, should emphasize the scientific knowledge. Should be emphasized amplitude of the area of the sports training, to have a mutual relationship between its basic elements.

Keywords: Sports training. Scientific knowledge. Cheers.

\footnotetext{
${ }^{1}$ Doutorando em Educação pela UFG. Mestre em Educação pela UFG. Professor do curso de Educação Física da Faculdade da Polícia Militar e Coordenador do Grupo de Estudos em Práticas Esportivas e Corporais (GEPEC). E-mail: wplima9gmail.com

${ }^{2}$ Mestre em Ciências da Saúde pela UNB. Coordenador do curso de Educação Física da Faculdade da Polícia Militar. Major da Polícia Militar do Estado de Goiás. E-mail: educadorreis@gmail.com

${ }^{3}$ Aluno do curso de Educação Física da Faculdade da Polícia Militar e membro do Grupo de Estudos em Práticas Esportivas e Corporais (GEPEC). E-mail: joao.bandeira@faculdadepm.edu.br
} 


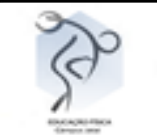

\section{INTRODUÇÃO}

Ao elaborar uma discussão, enfatizando o treinamento esportivo, torna-se necessário ressaltar que o mesmo, data de uma longa história e que mediante o decorrer dos tempos foi se aprimorando, desenvolvendo e consolidando como uma área do conhecimento científico.

Desde a Antiguidade, com os jogos olímpicos, até a Idade Moderna, com o culto ao corpo, no período pós-Idade Média, e, na contemporaneidade, com o status científico, a preocupação com a saúde, com o corpo sadio, o esporte e o treinamento sempre foram aspectos de destaque na sociedade.

De acordo com Böhme (2003), treinamento é um processo de ações complexas que tem como características: conteúdos, objetivos, métodos, organização e realização. Tais características com o intuito de trabalhar o atleta para que o mesmo esteja em sua melhor forma durante as competições.

Portanto, este artigo tem como objetivo central apresentar uma discussão sobre o treinamento esportivo, elucidando alguns de seus vários aspectos científicos, que contribuem com a evolução do desempenho esportivo do atleta e também de indivíduos que não são atletas, mas praticam esportes como forma de manter a saúde e o bem-estar. Importante destacar que o presente estudo também tem o intuito de contribuir com a pesquisa e a formação de profissionais da área da Educação Física.

\section{DESEVOLVIMENTO}

O presente estudo foi realizado mediante a revisão bibliográfica em livros e artigos científicos na área da Educação Física. O material encontrado foi estudado e analisado objetivando dar um suporte científico ao tema proposto.

\subsection{Princípios do treinamento}

Os Princípios do Treinamento Esportivo, segundo Dantas (1995), são os aspectos cuja observância irá diferenciar o trabalho feito, à base de ensaios e erros, do trabalho 


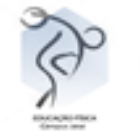

científico. São eles: Princípio da Individualidade Biológica; da Adaptação; da Sobrecarga; da Continuidade/Reversibilidade; e Interdependência, Volume x Intensidade. Cada qual com suas particularidades, todavia constata-se suas mútuas relações.

O Princípio da Individualidade Biológica, como o nome já descreve, enfatiza que cada ser humano é único, constituído por genótipo e fenótipo. O genótipo faz referência à carga genética transmitida à pessoa, ou seja, sua composição corporal, biotipo, altura máxima esperada, capacidades motoras e possível percentual de tipos de fibras musculares. O fenótipo versa sobre os fatores externos que modificam as características do indivíduo/atleta, somado ao indivíduo a partir do momento de seu nascimento, responsável por aspectos como habilidades motoras e esportivas. Destaca-se, nesse princípio, a individualização do treinamento, por grupos ou pessoas (DANTAS, 1995 e TUBINO, 1984).

Existem, portanto, questões biológicas e sociais que influenciam no desenvolvimento do indivíduo, seja ele atleta ou não. No caso do treinamento esportivo, os atletas recebem treinamentos específicos para cada área de sua atuação, por mais que seja uma equipe de voleibol, por exemplo, existem diferentes funções que necessitam ser trabalhas de modo diferenciado e esse trabalho é observado mediante aspectos principalmente genéticos como: altura, tipo de fibra, entre outros.

O Princípio da Adaptação está relacionado às diferentes capacidades de adaptação, mediante estímulos advindos dos exercícios e cargas de treinamento. Por exemplo: Estímulo - Organismo - Adaptação (DANTAS, 1995 e TUBINO, 1984). Importante destacar que o estímulo deve ser trabalhado de acordo com as capacidades ideais dos atletas. Portanto, vinculado a homeostase, sendo que o atleta necessita se adaptar ao treinamento, ou seja, todo estímulo provoca a característica de adaptação. Sem adaptação não há evolução e assim não há sobrevivência.

Esse princípio ressalta a importância de se saber dosar os diferentes estímulos que são exercidos sobre os atletas. Tais dosagens representam, de modo informal, acostumar o indivíduo à carga do treinamento de modo progressivo, evitando lesões, de modo que se faz necessária a correta prescrição do exercício.

Como precaução é necessário que o estímulo seja trabalho de modo crescente, ou seja, do mais leve e moderado para o mais pesado e intenso. Pois assim, pode desenvolver a 


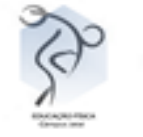

melhoria e evolução do rendimento. Já a falta de estímulo acarreta a estagnação e a não evolução no desempenho, enquanto o estímulo excessivo provoca lesões, como já dito, havendo assim, a má adaptação do indivíduo ao processo de treinamento. O desempenho, portanto, é o resultado de muitos anos de treinamento adequado, sistematizado, científico, havendo a necessidade de o treinamento induzir adaptações que melhorem o desempenho do indivíduo/atleta.

O Princípio da Sobrecarga diz respeito à supercompensação, que é a capacidade do organismo de restituir as energias gastas no treinamento, preparando-se para uma nova etapa de treino (TUBINO, 1984). A sobrecarga refere-se à observação de que um sistema ou tecido necessita receber uma quantidade além de carga de exercícios, para que de fato ocorra os efeitos do treinamento (DANTAS, 1995 e TUBINO, 1984).

As mudanças funcionais ocorrem somente quando as cargas de treinamento são suficientes para causar ativações no sistema biofisiológico, como por exemplo, é o caso da hipertrofia ou o emagrecimento. Assim sendo, o aumento de volume, no caso do princípio da sobrecarga é relacionada à quantidade ou duração de cada sessão de treinamento. A intensidade está relacionada ao aumento da velocidade de execução ou diminuição da quantidade de recuperação, por fim, a variação dos exercícios diz respeito à ordem de execução e mudança de metodologia dos treinos.

Portanto, quando se fala de volume e intensidade, assunto que será destacado mais adiante, está se referindo também e, sobretudo, à sobrecarga do treinamento.

O Princípio da Continuidade/Reversibilidade ressalta que as modificações induzidas pelo treinamento são passageiras. As características adquiridas por meio do treino perdem-se e retornam aos limites pré-treinamento após período de inatividade. Portanto, há sempre a necessidade de manutenção do treinamento, em níveis contínuos, para a manutenção de um estado de treinamento mais elevado (DANTAS, 1995).

Estes princípios estão nitidamente relacionados à Adaptação e a Sobrecarga. Dois aspectos fundamentais definem o princípio da continuidade: a interrupção e a duração do treinamento, prevendo a regularidade da prática do treinamento para o desenvolvimento das capacidades motoras em relação a saúde e ao esporte. 
O Princípio da Interdependência refere-se ao Volume x Intensidade. Baseando-se assim, na boa adequação de ambos. Como regra geral de segurança, propõem-se trabalhar primeiramente maior volume e, no decorrer do treinamento, ir aumentando a intensidade. $\mathrm{O}$ volume é importante para manter a estabilidade do rendimento, já a intensidade é importante para o aumento do rendimento.

O Volume refere-se à quantidade de treinamento, relacionada ao número de repetições, tempo de corrida, dias/semanas/meses de treinamento. A intensidade, diz respeito à qualidade do treinamento, manipulação de cargas, intervalos de repouso, velocidade da execução. Um organismo submetido ao trabalho muito intenso, somente poderá executá-lo em um curto espaço de tempo, e quando há um aumento desse espaço de tempo, é necessário diminuir a intensidade, valendo-se do pressuposto de que o importante é trabalhar de modo seguro, pesado e ideal. Desse modo, se a intensidade aumenta, o volume diminui e se o volume aumenta a intensidade diminui.

O Princípio da Especificidade está relacionado com o princípio da Individualidade Biológica, em um treinamento de alto nível. Portanto, o treinamento deve evidenciar as capacidades especificas do esporte praticado, mas também as particularidades dos atletas.

Enfim, com a exposição sobre os princípios do treinamento, que estão intrinsecamente relacionados uns aos outros, é imprescindível saber avaliar e controlar os níveis de treinamento e cargas, objetivando sempre a evolução do desempenho, de modo que não acarrete lesões nos atletas.

\subsection{Avaliação e controle}

A avaliação é um aspecto muito importante para o efetivo desempenho do atleta. A mesma compreende o diagnóstico das capacidades motoras envolvidas na modalidade esportiva alvo, se possível em condições que simulem a prática da atividade. Essas capacidades motoras, dizem respeito à força, à flexibilidade, à potência e à agilidade. Todas elas utilizadas, porém, em algumas modalidades esportivas, algumas são mais específicas que as outras. O importante é saber reconhece-las e trabalha-las adequadamente, para tanto o primeiro passo é a avaliação (MCARDLE, KATCH, KATCH, 2016). 
Entre os métodos de avaliação, encontram-se os testes, com seus protocolos sistematizados, visando aumentar a produtividade e os resultados, detectando, mesmo que sejam pequenas, alterações que ao final podem provocar grandes diferenças no desempenho. Por exemplo, além de protocolos, muito se tem utilizado vídeos para descrever melhor as capacidades motoras, principalmente em modalidades como: futebol, basquetebol, voleibol, entre outras.

Por se tratar de filmagens, os avanços tecnológicos permitem, hoje, avaliações mais completas, com outras variáveis para a compreensão do desempenho. Por exemplo, o uso de plataformas para verificar e quantificar, mediante cálculos matemáticos, avaliar diferentes modelos de treinamento da mecânica do movimento, enfatizando as diferentes forças internas: velocidade angular das articulações e sincronia dos movimentos em geral.

Outro exemplo é o uso de biopsias e da biologia molecular, objetivando avaliar o nível e o tipo de treinamento que está mais efetivo e gerando importantes adaptações e modificações positivas agudas e crônicas no organismo. Para, assim, haver o controle correto do treinamento.

O controle de treinamento diz respeito à prescrição do exercício, em uma relação de estímulo-resposta. Desse modo a resposta está associada à mudança e/ou alteração de um parâmetro fisiológico, decorrente da carga de treinamento, ou seja, pelo estímulo (MCARDLE, KATCH, KATCH, 2016).

Técnicos, preparadores físicos e cientistas têm buscado determinar parâmetros que sejam capazes de controlar a carga de treinamento, visando alterações positivas físicas e fisiológicas no desempenho esportivo. Como exemplo, pode ser citada a avaliação de oxigênio, concentração sanguínea de lactato, frequência cardíaca, percepção subjetiva do esforço. Cada qual com suas contribuições e limitações.

Determinar uma sessão de exercícios no organismo é muito importante, considerando a rotina de treinamento dos atletas, buscando evitar o excesso de treino, ou o esforço além do limite, minimizando a ocorrência de lesões.

Como exemplo, pode se considerar um profissional que atua com basquetebol, este sendo preparador físico de uma equipe, deve realizar seu treinamento de forma progressiva, organizando e planejando o desenvolvimento das capacidades físicas, sendo que, 


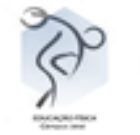

na fase inicial de treinamento é importante fortalecer todas as estruturas do organismo humano: orgânica, anatômica e fisiológica.

Para um trabalho de resistência, o preparador físico pode utilizar de exercícios para potencializar o funcionamento do sistema cardiovascular para posteriores cargas exigidas pelo ritmo de jogo do basquetebol, utilizando, por exemplo, treinamento intervalado, corridas, exercícios técnicos com bola e sem bola, mas sempre com atenção à capacidade cardiorrespiratória.

A flexibilidade e o treinamento de força são condições necessárias para melhores execuções de quaisquer movimentos em vários esportes, não somente do basquetebol, podem e devem ser inseridos de forma generalizada desde a iniciação esportiva, partindo de estímulos médios para serem aumentados de pouco a pouco, de acordo com as experiências vividas no trabalho com peso nas fases anteriores, ressaltando sempre as fundamentais contribuições do processo de avaliação e controle dos treinamentos. Portanto, ao tratar do trabalho de requisitos motores a serem desenvolvidos desde a iniciação esportiva, fala-se de especificidades e evolução das capacidades físicas, biológicas e psicológicas do indivíduo ao longo de tempo de treinamento.

\subsection{Da iniciação à competição: o treinamento de força para jovens, adultos e atletas}

Para Powers e Howley (2000) o treinamento de força tem o objetivo de provocar adaptações na musculatura esquelética através de sobrecargas. Esta sobrecarga, normalmente está relacionada a uma determinada porcentagem de uma repetição máxima, que se caracteriza pela maior carga que o indivíduo consegue realizar na realização de determinado exercício.

Destarte, a força muscular refere-se à força que um musculo pode gerar e é comumente expressa como uma repetição máxima (1RM). Já a resistência muscular se refere à capacidade de realização de contrações repetidas contra uma carga submáxima (POWERS e HOWLEY, 2000).

Em relação aos jovens e adultos, os objetivos são diferentes, bem como métodos a serem organizados e realizados. Entendendo suas dificuldades e possibilidades de melhoras, de acordo com o processo de treinamento, valoriza-se e se reconhece aspectos biofisológicos 


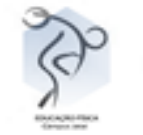

e psicossociais desses indivíduos, principalmente de jovens ${ }^{4}$, evitando o treinamento precoce, pulando fases de seu desenvolvimento.

Desse modo, é necessário ir além de reconhecer esses princípios do treinamento, deve-se tomar cuidado para não pular etapas e trabalhar os exageros do treinamento precoce em relação aos jovens. Essas etapas, dizem respeito ao próprio desenvolvimento natural do indivíduo, ressaltando fatores biofisiológicos e psicossociais. Como método de valorizar esses aspectos do desenvolvimento do indivíduo/jovem/atleta, o lúdico é fundamental, pois a partir do mesmo é possível estimular e desenvolver os requisitos motores e cognitivos dos praticantes.

Em relação aos adultos, devem-se enfatizar os princípios do treinamento, principalmente da adaptação e da sobrecarga. Pois, estes em suas mútuas relações, referem-se à capacidade adaptativa do indivíduo frente aos estímulos proporcionados pelos exercícios e cargas, ou seja, Estímulo, Organismo, Adaptação (DANTAS, 1995 e TUBINO 1984), como já enfatizado anteriormente.

No treinamento de força para atletas, além dos princípios treinamento, a periodização do treinamento deve vista e desenvolvida com atenção, havendo a compreensão de todo o tempo em que se pretende trabalhar com esse atleta. De acordo com Bompa (2001), periodização é o período, porção e divisão do tempo em pequenos segmentos mais fáceis de controlar, a fim de propiciar o desempenho máximo nas competições. É dividido em microciclo (menor estrutura do treinamento), mesociclo (conjunto de microciclos) e macrociclo (maior estrutura do treinamento, mensal, anual e plurianual). Portanto, a periodização é fundamental na fase de transição do atleta, da iniciação à competição; destacando-se, assim, a correta avaliação e controle do treinamento.

Para se trabalhar o treinamento, como exemplo, podem ser citados anilhas, halteres, aparelhos de academia, peso corporal, elásticos (teraband e garrotes). Portanto, o treinamento de força tem uma ampla utilização em aspectos relacionados à saúde, qualidade de vida, estética, tratamento de patologias, prevenção de doenças e lesões, treinamento de atletas de alto rendimento, melhoria na parte funcional (idosos).

\footnotetext{
${ }^{4}$ Entende-se jovem como adolescente, já adentrando a fase adulta.
} 
A classificação da ação muscular é dividida em Isométrica, que são exercícios que promovem contrações musculares, porém não executam movimentos, normalmente utilizada para manutenção da postura, funcionalmente estabilizando as articulações, e a Isotônica, que também promovem contrações musculares, no entanto proporciona-se movimento corporal, destacando a fase concêntrica e a fase excêntrica, muito utilizada para o desenvolvimento do volume muscular, potência e resistência muscular. Importante ressaltar que estas caraterísticas podem ser utilizadas para jovens, adultos e atletas, porém considerando suas especificidades.

A musculação melhora o desempenho de qualquer atleta. Quanto mais fibras musculares, mais rápida a ação do Sistema Nervoso Central e melhor o desempenho de vencer resistências em qualquer atleta.

A preparação de alto rendimento ${ }^{5}$ é indicada a ser iniciada após, o terceiro ano de treinamento, em pessoas que não são atletas, e, geralmente só começa após o quinto ano. Músculos treinados representa mais oxigênio por que, de acordo com MCardle, Katch, Katch (2016) há:

_ maior teor de mioglobina

_ maior número e tamanho de mitocôndrias

_ maior atividade e concentração de enzima

_ maior capacidade de oxidação de gordura

Em relação ao trabalho aeróbico, pode-se dizer que quanto maior a base aeróbica, maior o condicionamento do atleta. Quanto mais trabalha as valências físicas da aptidão física, maior o condicionamento do atleta e o volume máximo de oxigênio.

Portanto, o treinamento aeróbico Aumento da densidade capilar, aumento do volume sanguíneo, em fase de crescimento (aumento da caixa torácica, volume pulmonar e capacidade de difusão de $\mathrm{O} 2$ ), a melhoria geral da resistência implica maior resistência às infecções, ao calor, ao frio.

\section{CONSIDERAÇÕES FINAIS}

\footnotetext{
${ }^{5}$ Entende-se como preparação de alto rendimento, aquele treinamento que verdadeiramente irá cobrar um desempenho maior do indivíduo, sendo trabalho de modo rígido e sistematizado os treinos.
} 
Constata-se que existe uma mutua relação entre os princípios do treinamento esportivo, enfatizando-se que o caráter científico visa trabalhar os principais requisitos motores do indivíduo, sendo atleta ou não, no intuito de desenvolver a melhor capacidade biofisiológica do mesmo para um bom desempenho em competições ou para desempenhar suas atividades diárias com saúde e bem-estar.

Ao se trabalhar objetivando a efetiva melhoria no desempenho esportivo deve-se enfatizar o conhecimento científico, ressaltando a amplitude da área do treinamento esportivo, fazendo, assim, relação intrínseca entre avaliação e controle do treinamento, com seus outros aspectos fundamentais e científicos.

Enfim, como o próprio nome elucida, este é um estudo introdutório na área do treinamento esportivo e do exercício físico. Desse modo, indica-se a investigação, estudo e publicação de novos trabalhos que discorram sobre essa área tão importante para a formação do profissional de Educação Física, tendo sempre como base os conhecimentos científicos.

\section{REFERÊNCIAS}

BÖHME. M. S. Relações entre aptidão física, esporte e treinamento esportivo. 2003. Rev. Bra. Cie. Mov. Bra. v.11, n.3, 2003.

BOMPA, T. Periodização: Teoria e Metodologia do Treinamento. São Paulo. Phorte ed, 2001.

DANTAS, E. Prática da preparação física. 3ª ed. Rio de Janeiro, Shape, 1995.

MCARDLE,W.;KATCH,F.;KATCH,V. Fisiologia do Exercício - Energia, Nutrição e Desempenho Humano. 8.ed. Ed.Guanabara: Koogan, 2016.

POWER, S.; HOWLEY. E. Fisiologia do exercício: teoria e aplicação ao condicionamento e ao desempenho. $3^{\text {a }}$ ed. São Paulo, Manole, 2000.

TUBINO, M. Metodologia científica do treinamento esportivo. $3^{\text {a }}$ ed. São Paulo, Ibrasa, 1984. 